\title{
Complexity in the Role of Noise in Stochastic Systems
}

\section{R.K. Brojen Singh ${ }^{1 *}$ and B. Indrajit Sharma ${ }^{2}$}

${ }^{1}$ Centre for Interdisciplinary Research in Basic Sciences, Jamia Millia Islamia, New Delhi 110025, India

${ }^{2}$ Department of Physics, Assam University, Silchar-788011, Assam, India

\begin{abstract}
The role of noise in stochastic system could be constructive or destructive depending on the topology of the biochemical network, interaction mechanism and strength of the noise. The noise in genetic and chemical oscillators is studied using chemical Langevin equation and numerically using stochastic simulation algorithm to study the role of noise. The temporal behaviors of the variables in genetic oscillator are found to maintain their oscillatory nature at small system size limit $(V \leq 13 \pm 3)$, but above this the oscillatory behaviors get start destroying by getting transition from fluctuated limit cycle to no oscillation limit as $V \rightarrow \infty$. However, in chemical oscillator case, in the limit $V \rightarrow \infty$ the oscillatory behaviors of the variables get transition from fluctuated limit cycle (tending destroy oscillatory behavior) to normal limit cycle (maintain sustain oscillations). The noise parameter calculated as a function of $\mathrm{V}$ for genetic oscillator first slowly decrease and increases as $V$ increases. However the noise parameter in chemical oscillator decreases first $(V \leq 50 \pm 5)$ and then remains constant as $V$ increases. The calculated noise parameter via stochastic simulation algorithm is always found to be larger than that is obtained via chemical Langevin equation.
\end{abstract}

Keywords: Noise parameter; Genetic oscillator; Chemical oscillator; Master equation; Chemical Langevin equation

\section{Introduction}

The noise in stochastic system is due to random particle/ molecular interaction of the participating particles/molecules in the system (intrinsic noise) [1,2], and exchange of external fluctuations of the surrounding environment with the system such as thermal, environmental fluctuations (external noise) [3,4]. This noise, in general, is known to be a parameter that induces hindrance to the signal associated with dynamical variables of the system [5]. However, there are important constructive roles of noise in stochastic systems, for example, detection and amplification of weak noise, the phenomenon known as stochastic resonance [6,7], lifting of cellular expression at different distinct expression state [8], noise in gene expression can drive stochastic switching among such states $[9,10]$, noise induced stochastic phenotypic switching to different new level in living cells [11], noise induce generation of coherent motion [12], noise induced synchronization of stochastic systems [13] that can be found in intercircadian networks via environmental fluctuations [14-16] etc. This noise parameter is also being used as a means of synchronizing or correlating behaviors of a group of biological systems such as group of biological cells in multi-cellular organism or group of unicellular organisms, i.e. quorum sensing in a group of bacteria via external environmental fluctuation [17]. The study of the role of noise in realistic systems is of main interest especially in biological areas.

The complex processes in stochastic system can be well described by Master equation formalism (ME) which is based on every individual particle/molecular interaction involve that leads to decay or/and creation of particles/molecules in the system $[1,18,19]$. Since solving ME for complex system is difficult, there have been other simplified techniques to deal with processes in the system, for example, chemical Langevin equation (CLE) [20], noise induce deterministic equation (Langevin equation) [19,21] and Linear noise approximation [19,22]. Noise in the system described by ME can be well estimated using generating function technique $[18,19,23]$. The strength of the noise in the system depends on various parameters such as systems size $\mathrm{V}$, population of molecules accommodated in the systems $(\mathrm{N})$ and dynamical variables in the system $[1,20]$. For instance, the noise strength associated with single cell gene expression scales as $\mathrm{N}^{-1}$ of relative fluctuation amplitude [8] and in CLE it scales as $\mathrm{N}^{-1 / 2}$.

Noise, on the other hand, is a parameter similar to thermodynamics limit which can distinguish stochastic and deterministic systems, where stochastic system becomes deterministic when noise becomes negligible $[20,24]$. This parameter is an inherent property of most of the biological systems, and can be technically controlled by controlling the noise term introduced in the system or controlling the parameters such as system size, dynamical variables in the system etc. But in real practice, it is hard to control the internal noise which is already associated with molecular events taking place in real biological systems. However the role of noise could be of different varieties, different for different noise strengths and system properties. We study certain roles of noise in this work which could happen in natural system, and may unfold many interesting roles of noise in various natural systems. Our work is organized as follows. We first briefly describe stochastic model of biochemical network followed by the analysis of Chemical Langevin formalisms in materials and methods. We then pick up two examples namely genetic and chemical oscillator, and study the role of noise numerically as well as analytically which are presented in results and discussions. Finally some conclusions are drawn based on the results we obtained in this work.

\section{Materials and Methods}

The random interaction of molecules in a well stirred stochastic system leads the dynamics of the variables in the system to noise-driven stochastic process $[1,19,20]$. Out of two types of interaction, namely

*Corresponding author: R.K. Brojen Singh, Centre for Interdisciplinary Research in Basic Sciences, Jamia Millia Islamia, New Delhi-110025, India, Tel: 91126982408 (4498); Fax: 911-26981717 (4498); E-mail: brojen2k@yahoo.com

Received November 02, 2011; Accepted January 25, 2012; Published January 27, 2012

Citation: Brojen Singh RK, Indrajit Sharma B (2012) Complexity in the Role of Noise in Stochastic Systems. J Comput Sci Syst Biol 5: 016-023. doi:10.4172/ jcsb.1000086

Copyright: () 2012 Brojen Singh RK, et al. This is an open-access article distributed under the terms of the Creative Commons Attribution License,which permits unrestricted use, distribution, and reproduction in any medium, provided the original author and source are credited. 
Citation: Brojen Singh RK, Indrajit Sharma B (2012) Complexity in the Role of Noise in Stochastic Systems. J Comput Sci Syst Biol 5: 016-023. doi:10.4172/jcsb. 1000086

reacting and non-reacting collisions [25], reacting interaction, which involves decay and creation of molecular species, plays major roles in the stochastic system is responsible for various stochastic functions. The complex reactions in the system in real situation can be reduced to elementary reactions where few reactants are involved $[1,25]$. If we consider the state of the system at any instant of time $\mathbf{t}$ is defined by a configurational state vector, $\vec{X}(t)=\left[X_{1}(t), X_{2}(t), \cdots, X_{N}(t)\right]^{T}$, where $\mathbf{N}$ distinct molecular species are interacting via $\mathbf{M}$ elementary reaction channels in the system of the following type,

$$
\begin{aligned}
& a_{1 \mu} X_{1}+a_{2 \mu} X_{2}+\cdots+a_{N \mu} X_{N} \stackrel{k_{\mu}}{\rightarrow} \\
& b_{1 \mu} X_{1}+b_{2 \mu} X_{2}+\cdots+b_{N \mu} X_{N}
\end{aligned}
$$

where, $\mu$ is the reaction number index: $\mu=1, \ldots, \mathrm{N}, \mathrm{a}_{\mathrm{i} \mu}$ and $\mathrm{b}_{\mathrm{i} \mu}$ are coefficient to define stoichiometric matrix $v_{\mathrm{i} \mu}=\left(\mathrm{a}_{\mathrm{i} \mu}-\mathrm{b}_{\mathrm{i \mu}}\right)$, and $\mathrm{k}_{\eta}$ is the $\mu$ th macroscopic rate of reaction. The system evolves with various random reaction fired at random interval of time with decay or/and creation of particle/molecular species at any reaction event $[1,18,19,25]$ which leads to the change in configurational state of the system. This allows to define a configurational probability $P(\vec{X} ; t)$ as the probability to get this state change in an interval of time $[\mathrm{t}, \mathrm{t}+\mathrm{t}]$. Then the time evolution of configurational probability $P(\vec{X} ; t)$ obeys Chemical Master equation (ME) $[1,18,19]$ given by,

$$
\frac{\partial P(\overrightarrow{\boldsymbol{X}}, t)}{\partial t}=-\sum_{\overrightarrow{\boldsymbol{X}^{\prime}}} P(\overrightarrow{\boldsymbol{X}}, t) W_{\overrightarrow{\boldsymbol{X}} \rightarrow \overrightarrow{\boldsymbol{X}}^{\prime}}+\sum_{\overrightarrow{\boldsymbol{X}}} P\left(\overrightarrow{\boldsymbol{X}}^{\prime}, t\right) W_{\overrightarrow{\boldsymbol{X}}^{\prime} \rightarrow \overrightarrow{\boldsymbol{X}}}
$$

Where, $\{$ W $\}$ are transition probabilities to jump from one state, $\vec{X}$ of the molecular system to another state, $\overrightarrow{\boldsymbol{X}}$ during the time interval $[t, t+\Delta t]$. The ME in general provides detail stochastic description of chemical kinetics, but it is very difficult to solve for complex systems [19].

The chemical Langevin equation (CLE) formalism is one method to approximate ME to simpler continuous Markov type equations by keeping conditions which are applicable in natural systems [20], and the accuracy of this CLE is found to be more than those of other formalisms such as linear noise approximation [26]. The approximation can be done by allowing to define a function $Q(\vec{X}, \Delta t)$ as the number of a particular reaction fired during an interval of time $[\mathrm{t}, \mathrm{t}+\Delta \mathrm{t}]$ with $\Delta t>0$. This is followed by excellent approximations by imposing two conditions, firstly, imposing small $\Delta \mathrm{t}$ limit such that the values of propensity functions $\omega[\vec{X}(t)]$ of the reactions remain constant during $[\mathrm{t}, \mathrm{t}+\Delta \mathrm{t}]$, and secondly imposing large $\Delta \mathrm{t}$ limit which in turn leads to $\omega[\vec{X}(t)] \Delta t \gg 1$. These two conditions allow $\mathbf{Q}$ to approximate to statistically independent Poisson random variable and then the Poisson random variable is replaced by normal variable with the same mean and variance. Both the conditions are true in natural practice for large population limit. Then linearizing the normal variable, and defining $\begin{aligned} & \text { macroscopic molecular concentration vector, } \\ & \text { where } \mathbf{V} \text { is the systems size, we have general CLE, }\end{aligned} \quad\left\{\overrightarrow{x^{s}}(t)\right\}=\frac{1}{V} \vec{X}(t)$,

$$
\frac{d \vec{x}_{i}^{s}(t)}{d t}=F_{i}^{s}\left[\boldsymbol{\omega}\left(\boldsymbol{x}^{s}\right), v\right]+G_{i}\left[\boldsymbol{\omega}\left(\vec{x}^{s}\right), v, \mathbf{V}\right] \xi
$$

where, $\quad F_{i}^{s}=\sum_{i=1}^{M} v_{i j} \omega_{j}\left\{\vec{x}^{s}(\boldsymbol{t})\right\} \quad \mathrm{i}=1,2, \ldots, \mathrm{N} \quad$ is the macroscopic contribution term and $G_{i}=\frac{1}{\sqrt{V}} \sum_{i=1}^{M} v_{i j}\left[\grave{\mathrm{u}}_{\mathrm{i}}\left\{\vec{x}^{s}(\mathbf{t})\right\}\right]^{1 / 2}$ is the stochastic contribution term to the dynamics. $\xi_{i}=\lim _{d t \rightarrow 0} N_{i}(0,1) / \sqrt{d t}$ is uncorrelated, statistically independent random noise parameters which satisfy $\xi_{i}(t) \xi_{j}\left(t^{\prime}\right)=\delta_{i j} \delta\left(t-t^{\prime}\right)$. The stochastic CLE (2) will become deterministic equation when $\xi \rightarrow 0$, and equation (2) becomes,

$$
\frac{d \vec{x}_{i}^{d}(t)}{d t}=F_{i}^{s}\left[\omega\left(x^{d}\right), v\right]
$$

where, $F_{i}^{s}=\sum_{i=1}^{M} v_{i j} \omega_{j}\left\{\vec{x}^{d}(\boldsymbol{t})\right\}$ is the deterministic function. If the stochastic and deterministic variables obtained from equations (3) and (4) are given by, $\vec{x}^{s}(\boldsymbol{t})=\left[\boldsymbol{x}_{1}^{s}(\boldsymbol{t}), \boldsymbol{x}_{2}^{s}(\boldsymbol{t}), \cdots, \boldsymbol{x}_{N}^{s}(\boldsymbol{t})\right]^{T}$ and $\vec{x}^{d}(t)=\left[x_{1}^{d}(t), x_{2}^{d}(t), \ldots, x_{N}^{d}(t)\right]^{T}$ respectively then the noise parameter can be defined by $\vec{\eta}_{i}(t)=\vec{x}_{i}^{s}(t)-U_{i} \vec{x}_{i}^{d}(t)$, where $\vec{U}=\left[U_{1}\left(\vec{x}^{d}\right), U_{2}\left(\vec{x}^{d}\right), \cdots, U_{N}\left(\vec{x}^{d}\right),\right]^{T}$. The reason could be the stochastic variables are noise-induced variables, whereas deterministic variables are approximately noise free variables and non-linearity of both the CLE and deterministic equations. The time evolution of $\vec{\eta}_{i}(t)$ can be expressed by,

$$
\begin{aligned}
& \frac{d \vec{\eta}_{i}(t)}{d t}=\left[F_{i}^{s}\left\{\boldsymbol{\omega}\left(\boldsymbol{x}^{s}\right), v\right\}-\boldsymbol{U}_{i} F_{i}^{d}\left\{\boldsymbol{\omega}\left(\boldsymbol{x}^{d}\right), v\right\}\right]+ \\
& G_{i}\left[\boldsymbol{\omega}\left(\vec{x}^{s}\right), v, \mathbf{V}\right] \xi-\vec{x}_{i}^{d} \frac{d U_{i}}{d t}
\end{aligned}
$$

The dynamics of $\vec{\eta}_{i}(t)$ could be different for different stochastic systems and dependent on various parameters such as $\vec{x}^{s}, \boldsymbol{V}, \xi$ etc. The role of intrinsic noise in stochastic system could be different depending on the nature of interaction of the molecular species, network topology and system size. The steady state solution in $\mu_{, s, m, \sigma} \mathrm{can}$ be obtained from equation (5) as,

$$
\begin{aligned}
& F_{i}^{*_{s}}\left[\boldsymbol{\omega}\left(\boldsymbol{x}^{* s}\right), v\right]=U_{i} F_{i}^{d}\left[\boldsymbol{\omega}\left(\boldsymbol{x}^{* d}\right),\right]- \\
& G_{i}\left[\boldsymbol{\omega}\left(\vec{x}^{* s}\right), v, \boldsymbol{V}\right]+\vec{x}_{i}^{d} \frac{d \boldsymbol{U}_{\boldsymbol{i}}}{d t}
\end{aligned}
$$

where, $F_{i}^{*_{s}}, F_{i}^{d}, \boldsymbol{x}^{*_{s}}$ and $\boldsymbol{x}^{*_{d}}$ are steady state solutions. If $\vec{U}$ is found to be constant then $\boldsymbol{x}^{* \boldsymbol{d}}$ in stochastic CLE is proportional to the corresponding $\boldsymbol{x}^{* d}$ in deterministic system.

We used standard stochastic simulation algorithm due to Gillespie [1] to simulate the biochemical reaction network model of stochastic system. The algorithm systematically takes into account each and every reaction events to allow transitions from one state to another along the trajectory of the variables by defining a joint probability density function $\Omega(\tau, \mu)=\Pi(\tau) \chi(\mu)$. The reaction time and reaction number fired at that time can be estimated computationally by generating two uniform independent random numbers $r_{1}$ and $r_{2}$ to identify $\tau$ by $\tau=\frac{1}{\sum_{i} \omega_{i}} \ln \left[\frac{1}{r_{1}}\right]$ and reaction number $\mu$ by $f_{\mu}=r_{2} \sum_{i} \omega_{i}$ by imposing the relation $\sum_{i=1}^{\mu} \omega_{i} \leq f_{\mu}<\sum_{i=1}^{\mu+1} \omega_{i}$. Incorporating these two random numbers corresponding to the probabilities of finding reaction time and reaction number fired, the algorithm systematically calculate the state vector as a function of reaction time and one can find the temporal trajectory of the state vector.

The system size in the SSA can be associated by $V=\frac{\bar{V}}{N_{A}}$, where 
Citation: Brojen Singh RK, Indrajit Sharma B (2012) Complexity in the Role of Noise in Stochastic Systems. J Comput Sci Syst Biol 5: 016-023. doi:10.4172/jcsb.1000086

$N_{A}$ is Avogadro's number and $\bar{V}$ is average system size. The factor $\mathrm{V}$ is incorporated in the SSA from the relation which connect microscopic transition rate or propensity function $\left(\mathrm{c}_{\mathrm{ip}}\right)$ with macroscopic rate constant $\left(\mathrm{k}_{\mathrm{i \mu}}\right), \mathrm{c}_{\mathrm{i} \mu}=\mathrm{k}_{\mathrm{i} \mu} \mathrm{V}^{1-\mathrm{v}}{ }_{\mathrm{i \mu}}[1,27]$.

\section{Results and Discussion}

We study two stochastic models, namely genetic and chemical oscillators in which the roles of noise could be contrast. We concentrate mainly on the role of the noise in these oscillators by implementing the methods describe above and its dependence on various parameters. We then present numerical results using stochastic simulation algorithm.

\section{Genetic oscillator}

We use the minimal reaction model of Vilar et al. [28] based on the auto regulation of the two genes, an activator of $\mathrm{A}$ protein and a repressor of $\mathrm{R}$ protein: $\mathrm{A}$ acts as positive regulator binding to $\mathrm{A}$ promoter and $\mathrm{R}$ to increase their transcription rate, whereas $\mathrm{R}$ acts as negative regulator by sequestering $A[28,29]$. The regulatory mechanisms of A and $\mathrm{R}$ incorporate activator genes, $D_{a}^{\prime}$ and $D_{a}$ and repressor genes, $D_{r}^{\prime}$ and $D_{r}$, which involves mRNA of A and R, $\mathrm{M}_{\mathrm{a}}$ and $\mathrm{M}_{\mathrm{r}^{\circ}} \mathrm{C}$ corresponds to the inactivated complex formed by $\mathrm{A}$ and $\mathrm{R}$. The detail description of the biochemical reaction network is given in Table 1. This genetic oscillator model could able to generate 24 hour period of oscillation in $\mathrm{A}$ and $\mathrm{R}$ proteins and therefore can be taken as circadian clock.

The trajectory of the population variables involve in the genetic oscillator model as a function of time can be traced by identifying the state vector $\vec{X}(t)=\left[X_{1}, X_{2}, \cdots, X_{9}\right]^{T}$ at any instant of time t. where, $X_{2}=D_{a}^{\prime}, \mathrm{X}_{3}=\mathrm{D}_{\mathrm{r}}, X_{4}=D_{r}^{\prime}, X_{5}=M_{5}, X_{6} M_{r}, X_{7}=C, X_{8}=A, X_{9}=R$.. The Master equation of the model based on the reaction network given in Table 1 is given by,

$$
\begin{aligned}
& \frac{\partial P\left(D_{a}, D_{a}^{\prime}, \ldots, R ; t\right)}{\partial t} \\
& =\theta_{a}\left(D_{a}^{\prime}+1\right) P\left(D_{a}-1, D_{a}^{\prime}+1, \ldots, ; t\right)+\gamma_{a}\left(D_{a}+1\right)(A+1) \\
& P\left(D_{a}+1, D_{a}^{\prime}-1, A+1, \ldots, ; t\right)+\theta_{r}\left(D_{r}^{\prime}+1\right) P\left(D_{r}-1, D_{r}^{\prime}+1, \ldots, ; t\right)+ \\
& \gamma_{r}\left(D_{r}+1\right)(A+1) P\left(D_{r}+1, D_{r}^{\prime}-1, A+1, \ldots, ; t\right)+ \\
& \alpha_{r}^{\prime} D_{r}^{\prime} P\left(M_{r}-1, \ldots ; t\right)+\alpha_{r} D_{r} P\left(M_{r}-1, \ldots ; t\right)+\delta_{m r}\left(M_{r}+1\right) \\
& P\left(M_{r}+1, \ldots ; t\right)+\alpha_{a}^{\prime} D_{a}^{\prime} P\left(M_{a}-1, \ldots ; t\right)+\alpha_{a} D_{a} P\left(M_{a}-1, \ldots ; t\right) \\
& +\delta_{m a}\left(M_{a}+1\right) P\left(M_{a}+1, \ldots ; t\right)+\beta_{r} M_{r} P(R-1, \ldots ; t)+ \\
& \delta_{r}(R+1) P(\ldots, R+1 ; t)+\delta_{a}(C+1) P(\ldots, C+1, R-1 ; t) \\
& +\beta_{a} M_{a} P(A-1, \ldots ; t)+\theta_{a} D_{a}^{\prime} P(A-1, \ldots, ; t)+\theta_{r} D_{r}^{\prime} P(A-1, \ldots, ; t) \\
& +\delta_{a}(A+1) P(A+1, \ldots ; t)+\gamma_{c}(R+1)(A+1) P(\ldots, R+1, A+1, C-1 ; t)- \\
& \left(\theta_{a} D_{a}^{\prime}+\gamma_{a} D_{a} A+\theta_{r} D_{r}^{\prime}+\gamma_{r} D_{r} A+\alpha_{r}^{\prime} D_{r}^{\prime}+\alpha_{r} D_{r}+\delta_{m r} M_{r}+\right. \\
& \alpha_{a}^{\prime} D_{a}^{\prime}+\alpha_{a} D_{a}+\delta_{m a} M_{a}+\beta_{r} M_{r}+\delta_{r} R+\delta_{a} C \\
& \left.+\beta_{a} M_{a}+\theta_{a} D_{a}^{\prime}+\theta_{r} D_{r}^{\prime}+\delta_{a} A+\gamma_{c} R A\right) P\left(D_{a}, D_{a}^{\prime} \ldots, R ; t\right)
\end{aligned}
$$

\begin{tabular}{|c|c|c|c|c|}
\hline $\begin{array}{l}\text { S. } \\
\text { No. }\end{array}$ & Reactions & Description of the reactions & $\begin{array}{l}\text { Transition } \\
\text { rate }\end{array}$ & $\begin{array}{l}\text { Rate } \\
\text { constant } \\
\text { values }\end{array}$ \\
\hline 1. & $D_{a}^{\prime} \underline{\theta_{a}} D_{a}$ & $\begin{array}{l}\text { Conversion of activator genes with } \\
\text { A to activator genes without } \mathbf{A} \text {. }\end{array}$ & $\omega_{1}=\theta_{a} D_{a}^{\prime}$ & $\alpha_{a}=50$ \\
\hline 2. & $A+D_{a} \underline{\gamma_{a}} D_{a}^{\prime}$ & $\begin{array}{c}\text { Activation of activator gene } \\
\text { without } \mathbf{A} \text { to form activator gene } \\
\text { with } \mathbf{A} \text {. }\end{array}$ & $\omega_{2}=\gamma_{a} \frac{A D_{a}}{V}$ & $\alpha_{a}^{\prime}=500$ \\
\hline 3. & $D_{r}^{\prime} \theta_{r} D_{r}$ & $\begin{array}{l}\text { Conversion of repressor promoter } \\
\text { with } \mathbf{R} \text { to repressor promoter } \\
\text { without } \mathbf{R} \text {. }\end{array}$ & $\omega_{3}=\theta_{r} D_{r}^{\prime}$ & $\alpha_{r}=0.01$ \\
\hline 4. & $A+D_{r} \underline{\gamma}_{\underline{r}} D_{r}^{\prime}$ & $\begin{array}{l}\text { Activation of repressor promoter } \\
\text { without } \mathbf{R} \text { to form repressor } \\
\text { promoter with } \mathbf{R} \text {. }\end{array}$ & $\omega_{4}=\gamma_{r} \frac{A D_{r}}{V}$ & $\alpha_{r}^{\prime}=50$ \\
\hline 5. & $\varnothing \underline{\alpha_{a}^{\prime} D_{a}^{\prime} M_{a}}$ & $\begin{array}{c}\text { Creation of mRNA of activator } \\
\text { gene } \mathbf{A} \text { activated by activator } \\
\text { gene with } \mathbf{A} \text {. }\end{array}$ & $\omega_{5}=\alpha_{a}^{\prime} D_{a}^{\prime}$ & $\beta_{a}=50$ \\
\hline 6. & $\varnothing \underline{\alpha_{a} D_{a}} M_{a}$ & $\begin{array}{c}\text { Creation of mRNA of activator } \\
\text { gene } \mathbf{A} \text { activated by activator } \\
\text { gene without } \mathbf{A} \text {. }\end{array}$ & $\omega_{6}=\alpha_{a} D_{a}$ & $\beta_{r}=5$ \\
\hline 7. & $M_{a} \underline{\delta_{a} M_{a} \varnothing}$ & $\begin{array}{c}\text { Degradation of mRNA of activator } \\
\text { gene } \mathbf{A} \text {. }\end{array}$ & $\omega_{7}=\delta_{a} M_{a}$ & $\delta_{M A}=10$ \\
\hline 8. & $\varnothing \alpha_{r}^{\prime} D_{r}^{\prime} M_{r}$ & $\begin{array}{l}\text { Creation of mRNA of repressor } \\
\text { promoter } \mathbf{R} \text { activated by repressor } \\
\text { promoter with } \mathbf{R} \text {. }\end{array}$ & $\omega_{8}=\alpha_{r}^{\prime} D_{r}^{\prime}$ & $\delta_{M R}=0.5$ \\
\hline 9. & $\varnothing \alpha_{r} D_{r} M_{r}$ & $\begin{array}{l}\text { Creation of mRNA of repressor } \\
\text { promoter } \mathbf{R} \text { activated by repressor } \\
\text { promoter without } \mathbf{R} \text {. }\end{array}$ & $\omega_{9}=\alpha_{r} D_{r}$ & $\delta_{a}=1$ \\
\hline 10. & $M_{r} \underline{\delta_{r} M_{r} \varnothing}$ & $\begin{array}{l}\text { Degradation of mRNA of } \\
\text { repressor promoter } \mathbf{R} \text {. }\end{array}$ & $\omega_{10}=\delta_{r} M_{r}$ & $\delta_{r}=0.2$ \\
\hline 11. & $\varnothing \underline{\beta_{r} M_{r} R}$ & $\begin{array}{c}\text { Creation of repressor promoter } \mathbf{R} \\
\text { induced by mRNA of repressor } \\
\text { promoter } \mathbf{R} \text {. }\end{array}$ & $\omega_{11}=\beta_{r} M_{r}$ & $\gamma_{a}=1$ \\
\hline 12. & $R \delta_{r} R \varnothing$ & $\begin{array}{l}\text { Degradation of repressor } \\
\text { promoter } \mathbf{R} .\end{array}$ & $\omega_{12}=\delta_{r} R$ & $\gamma_{r}=1$ \\
\hline 13. & $C \underline{\delta_{a} C R}$ & $\begin{array}{c}\text { Creation of repressor promoter } \\
\mathbf{R} \text { by } \mathbf{R} \text {. }\end{array}$ & $\omega_{13}=\delta_{a} C$ & $\gamma_{c}=2$ \\
\hline 14. & $\varnothing \underline{\beta_{a} M_{a} A}$ & $\begin{array}{c}\text { Creation of activator gene } \mathbf{A} \\
\text { induced by mRNA of activator } \\
\text { gene } \mathbf{A} \text {. }\end{array}$ & $\omega_{14}=\beta_{a} M_{a}$ & $\theta_{a}=50$ \\
\hline 15. & $\varnothing \underline{\theta_{a} D_{a}^{\prime} A}$ & $\begin{array}{l}\text { Creation of activator gene } \mathbf{A} \\
\text { induced by } D_{a}^{\prime} \text {. }\end{array}$ & $\omega_{15}=\theta_{a} D_{a}^{\prime}$ & $\theta_{r}=100$ \\
\hline 16. & $\varnothing \underline{\theta_{r} D_{r}^{\prime} A}$ & $\begin{array}{l}\text { Creation of activator gene } \mathbf{A} \\
\text { induced by } D_{r}^{\prime} \text {. }\end{array}$ & $\omega_{16}=\theta_{r} D_{r}^{\prime}$ & \\
\hline 17. & $A \stackrel{\delta_{a} A \varnothing}{\longrightarrow}$ & Degradation of activator gene $\mathbf{A}$. & $\omega_{17}=\delta_{a} A$ & \\
\hline 18. & $A+D_{r} \gamma_{c} A R C$ & $\begin{array}{l}\text { Activation of activator gene } \mathbf{A} \text { to } \\
\text { form complex } \mathbf{C} \text { with } D_{r}\end{array}$ & $\omega_{18}=\gamma_{c} A R$ & \\
\hline
\end{tabular}

The microscopic variables are connected to macroscopic variables $\operatorname{via}\{\vec{x}(t)\}=\frac{1}{v} \vec{X}(t)$. This Master equation of the genetic oscillator model can be reduced to a set of CLE and can be obtained following
Table 1: The biochemical reaction network of genetic oscillator, description of the reactions, transition rates and values of the rate constants used in the simulation [27].

Gillespie's approach [19] as given below,

$$
\begin{aligned}
& \frac{d x_{1}(t)}{d t}=\theta_{A} x_{2}-\gamma_{A} x_{1} x_{8}+ \\
& \frac{1}{\sqrt{V}}\left[\sqrt{\theta_{A} x_{2} \xi_{1}}-\sqrt{\gamma_{A} x_{1} x_{8} \xi_{2}}\right] \\
& \frac{d x_{2}(t)}{d t}=\gamma_{A} x_{1} x_{8}-\theta_{A} x_{1}+ \\
& \frac{1}{\sqrt{V}}\left[\sqrt{\gamma_{A} x_{1} x_{8} \xi_{3}}-\sqrt{\theta_{A} x_{2} \xi_{4}}\right]
\end{aligned}
$$




$$
\begin{aligned}
& \frac{d x_{3}(t)}{d t}=\theta_{R} x_{4}-\gamma_{R} x_{3} x_{8}+ \\
& \frac{1}{\sqrt{V}}\left[\sqrt{\theta_{R} x_{4} \xi_{5}}-\sqrt{\gamma_{R} x_{3} x_{8} \xi_{6}}\right] z \\
& \frac{d x_{4}(t)}{d t}=\gamma_{R} x_{3} x_{8}-\theta_{R} x_{4}+ \\
& \frac{1}{\sqrt{V}}\left[\sqrt{\gamma_{R} x_{3} x_{8} \xi_{7}}-\sqrt{\theta_{R} x_{4} \xi_{8}}\right] \\
& \frac{d x_{5}(t)}{d t}=\alpha_{A}^{\prime} x_{2}+\alpha_{A} x_{1}-\delta_{M A} x_{5}+ \\
& \frac{1}{\sqrt{V}}\left[\sqrt{\alpha_{A}^{\prime} x_{2} \xi_{9}}+\sqrt{\alpha_{A} x_{1} \xi_{10}}-\sqrt{\delta_{M A} x_{5} \xi_{11}}\right] \\
& \frac{d x_{6}(t)}{d t}=\alpha_{R}^{\prime} x_{4}+\alpha_{R} x_{3}-\delta_{M R} x_{6}+ \\
& \frac{1}{\sqrt{V}}\left[\sqrt{\alpha_{R}^{\prime} x_{4} \xi_{12}}+\sqrt{\alpha_{R} x_{3} \xi_{13}}-\sqrt{\delta_{M R} x_{6} \xi_{14}}\right] \\
& \frac{d x_{7}(t)}{d t}=\gamma_{C} x_{8} x_{9}-\delta_{A} x_{7}+ \\
& \frac{1}{\sqrt{V}}\left[\sqrt{\gamma_{C} x_{8} x_{9} \xi_{15}}-\sqrt{\delta_{A} x_{7} \xi_{16}}\right] \\
& \frac{d x_{8}(t)}{d t}=\beta_{A} x_{5}+\theta_{A} x_{2}+ \\
& \theta_{R} x_{4}-x_{8}\left(\gamma_{A} x_{1}+\gamma_{R} x_{3}+\gamma_{C} x_{9}+\gamma_{C} x_{9}\right) \\
& +\frac{1}{\sqrt{V}}\left[\begin{array}{l}
\sqrt{\beta_{A} x_{5} \xi_{17}}+\sqrt{\theta_{A} x_{2} \xi_{18}}+\sqrt{\theta_{R} x_{4} \xi_{19}}- \\
\sqrt{\gamma_{A} x_{1} x_{8} \xi_{20}}-\sqrt{\gamma_{R} x_{3} x_{8} \xi_{21}}-\sqrt{\gamma_{C} x_{9} x_{8} \xi_{22}} \\
-\sqrt{\gamma_{C} x_{9} x_{8} \xi_{23}}
\end{array}\right] \\
& \frac{d x_{9}(t)}{d t}=\beta_{R} x_{6}-\gamma_{C} x_{8} x_{9}+\delta_{A} x_{7}-\delta_{R} x_{9}+\frac{1}{\sqrt{V}} \\
& {\left[\sqrt{\beta_{R} x_{6} \xi_{24}}-\sqrt{\gamma_{C} x_{8} x_{9} \xi_{25}}+\sqrt{\delta_{A} x_{7} \xi_{26}}-\sqrt{\delta_{R} x_{9} \xi_{27}}\right]}
\end{aligned}
$$

where, $\mathbf{V}$ is the systems size. The constraints to be satisfied by the activator and repressor genes are given by, $x_{1}+x_{2}=1$ and $x_{3}+x_{4}=1$. The CLEs reduce to deterministic equations when $V \rightarrow \infty, \xi_{i} \rightarrow 0$, and noise term in stochastic system described by CLEs is proportional to $V^{-1 / 2}$. The steady state solutions of the CLEs can be obtained by imposing the condition, $\left\{\frac{d x_{i}(t)}{d t}\right\}=0, i=1,2, \ldots, 9$. For deterministic equations the steady state solutions for $x_{7}^{* d}, x_{8}^{* d}$ and $x_{9}^{* d}$ and are given by,

$$
\begin{aligned}
& x_{7}^{*_{d} d} \sim \frac{\gamma_{R} \gamma_{C} \beta_{R} \alpha_{R}^{\prime} v^{2}}{\delta_{A} \delta_{R} \delta_{M R} \varphi^{2}} \frac{\left(1+\frac{\varphi u}{v^{2}}\right)^{2}}{\theta_{R}+\frac{\gamma_{R} v}{\varphi}\left(1+\frac{\varphi u}{v^{2}}\right)} \\
& x_{9}^{* d} \sim \frac{v}{\varphi}\left(1+\frac{\varphi u}{v^{2}}\right)
\end{aligned}
$$

$$
\begin{aligned}
& x_{9}^{*_{d}} \sim \frac{\beta_{R}}{\delta_{R} \delta_{M R}}\left[\frac{\frac{\gamma_{R} \alpha_{R}^{\prime} v}{\varphi}\left(1+\frac{\varphi u}{v^{2}}\right)}{\theta_{R}+\frac{\gamma_{R} v}{\varphi}\left(1+\frac{\varphi u}{v^{2}}\right)}+\frac{\theta_{R} \alpha_{R}}{\theta_{R}+\frac{\gamma_{R} v}{\varphi}\left(1+\frac{\varphi u}{v^{2}}\right)}\right] \\
& \text { Where, } u=\frac{\beta_{A} \theta_{A}\left(\alpha_{A}-\alpha_{A}^{\prime}\right)}{\gamma_{A} \delta_{M A}}-\frac{\gamma_{C} \beta_{R} \theta_{R}^{2}\left(\alpha_{R}^{\prime}-\alpha_{R}\right)}{\delta_{R} \delta_{M R} \gamma_{R}^{2}}, \\
& v=\frac{\beta_{A} \alpha_{A}^{\prime}}{\delta_{M A}}+\frac{\gamma_{C} \beta_{R} \theta_{R}\left(\alpha_{R}^{\prime}-\alpha_{R}\right)}{\delta_{R} \delta_{M R} \gamma_{R}}
\end{aligned}
$$

and $\varphi=\delta_{A}+\frac{\gamma_{C} \beta_{R} \alpha_{R}^{\prime}}{\delta_{R} \delta_{M R}}$ are constants. The above steady state solutions are obtained by taking terms upto of the order of $\mathrm{A}^{-2}$ and keeping the condition, discriminent $\geq 0$ which gives the condition:

$$
\alpha_{A}^{\prime}-\alpha_{A} \leq \frac{q^{2}}{g^{2} p},
$$

where, $p=\varphi \frac{\beta_{A} \theta_{A}}{\gamma_{A} \delta_{M A}}, q=\varphi \frac{\gamma_{C} \beta_{R} \theta_{R}^{2}}{\delta_{R} \delta_{M R} \gamma_{R}^{2}}$

and $g=\frac{\gamma_{C} \beta_{R} \theta_{R}}{\delta_{R} \delta_{M R} \gamma_{R}}$

Now the steady state solutions $x_{7}^{*_{s}}, x_{8}^{*_{s}}$ and $x_{9}^{*_{s}}$ in stochastic system can be obtained by solving the CLEs in equations (7)-(15) and keeping terms upto of the order of $\left[x_{7}^{*_{s}}\right]^{-2}$. The results are given by,

$$
\begin{aligned}
& x_{7}^{*_{s}}\left(x_{7}^{* d}, V, \xi_{1}\right) \sim U_{1} x_{7}^{*^{* d}}\left[1+\frac{\xi_{1}}{\sqrt{V}}\left(\frac{\Gamma+\Lambda}{x_{8}^{* d} x_{9}^{* d}}\right)\right] \\
& x_{8}^{*_{s}}\left(x_{8}^{* d}, V, \xi_{2}\right) \sim U_{2} x_{8}^{*^{d d}}\left[1-\frac{\xi_{2}}{\sqrt{V}} \Lambda\right] \\
& x_{9}^{*_{s}}\left(x_{9}^{* d}, V, \xi_{3}\right) \sim U_{3} x_{9}^{* d}+\frac{3}{\sqrt{V}} \Gamma
\end{aligned}
$$

Where, $\left\{\xi_{i}\right\}$ of the terms involved in deriving certain equation are taken to be the same to simplify the expression. The vector $\vec{U}$ is found to be $\vec{U}=\left[U_{1}, U_{2}, U_{3}\right]^{T}=\left[6, \frac{8}{5}, \frac{7}{2}\right]^{T}$. The constant expressions $\Lambda$ and $\Gamma$ are given by,

$$
\begin{aligned}
& \Lambda=\frac{I+\frac{v H}{\varphi}+\varphi J-u H-\frac{u \varphi I}{v}}{v^{2}+\varphi u} \\
& \Gamma=\frac{\beta_{R}}{\delta_{R} \delta_{M R}} \\
& {\left[\alpha_{R}^{\prime}+\sqrt{\frac{\alpha_{R} \theta_{R}}{\frac{v \gamma_{R}}{\varphi}\left(1+\frac{\varphi u}{v^{2}}\right)}}+\frac{\alpha_{R}^{\prime} \sqrt{\theta_{R}}}{\frac{v \gamma_{R}}{\varphi}\left(1+\frac{\varphi u}{v^{2}}\right)}+\sqrt{V} \frac{\alpha_{R}^{\prime}}{\delta_{R}} \sqrt{\frac{\beta_{R}}{\delta_{M R}}}\right]}
\end{aligned}
$$

And,

$$
H=\frac{\gamma_{C} \beta_{R}}{\delta_{R} \delta_{M R}} \sqrt{\alpha_{R}^{\prime}}+\sqrt{V} \frac{\delta_{R} \alpha_{R}^{\prime} \sqrt{\beta_{R}}}{\delta_{R} \delta_{M R}}
$$


Citation: Brojen Singh RK, Indrajit Sharma B (2012) Complexity in the Role of Noise in Stochastic Systems. J Comput Sci Syst Biol 5: 016-023. doi:10.4172/jcsb.1000086

\begin{tabular}{|c|c|c|c|}
\hline S. No. & Reactions & Transition rate & Rate constant values \\
\hline 1. & $D_{a}^{\prime} \underline{\theta_{a}} D_{a}$ & $\omega_{1}=\theta_{a} D_{a}^{\prime}$ & $4 \times 10^{-3}$ \\
\hline 2. & $X+Y \underset{\rightarrow}{k_{2} B}$ & $\omega_{2}=k_{2} \frac{X Y}{V}$ & 0.1 \\
\hline 3. & $C+X \underset{\rightarrow}{k_{3}} 2 X+Z$ & $\omega_{2}=k_{3} C X$ & $1.04 \times 10^{-2}$ \\
\hline 4. & $\underset{\rightarrow}{2 X k_{4} D}$ & $\omega_{2}=k_{4} \frac{1}{2 V} X(X-1)$ & $1.6 \times 10^{-2}$ \\
\hline 5. & $E+Z \underset{\rightarrow}{k_{5}} Y$ & $\omega_{2}=k_{5} E Z$ & $1.3 \times 10^{-2}$ \\
\hline
\end{tabular}

Table 2: The biochemical reaction channels involved in chemical oscillator (oregonator), microscopic transition rates and the values of the macroscopic rate constants used in our simulations in both deterministic and stochastic systems.

$$
\begin{aligned}
& I=\sqrt{\alpha_{R}^{\prime}}+\sqrt{\frac{\beta_{R} \alpha_{R}^{\prime}}{\delta_{M A}}}+\frac{\gamma_{C} \beta_{R} \theta_{R} \sqrt{\alpha_{R}^{\prime}}}{2 \delta_{R} \delta_{M R} \gamma_{R}}- \\
& \frac{\gamma_{C} \beta_{R} \theta_{R}\left(\alpha_{R}^{\prime}-\alpha_{R}\right)}{\delta_{R} \delta_{M R} \gamma_{R}}+\frac{\gamma_{C} \theta_{R}\left(\alpha_{R}^{\prime}-\alpha_{R}\right)}{\delta_{R} \gamma_{R}} \sqrt{\frac{\beta_{R}}{\delta_{M R}}} \\
& J=\frac{\theta_{A} \sqrt{\alpha_{A}^{\prime}}}{2 \gamma_{A}}+\sqrt{\frac{\beta_{A} \alpha_{A}^{\prime}}{\delta_{M A}}} \frac{\theta_{A}\left(\alpha_{A}^{\prime}-\alpha_{A}\right)}{2 \gamma_{A}}- \\
& \frac{\theta_{A}\left(\alpha_{A}^{\prime}-\alpha_{A}\right)}{\gamma_{A}}-\frac{\gamma_{C} \beta_{R} \theta_{R}^{2}\left(\alpha_{R}^{\prime}-\alpha_{R}\right)}{2 \delta_{R} \delta_{M R} \gamma_{R}^{2}}
\end{aligned}
$$

The noise terms in steady state solutions in equations (19), (20) and (21) are dependent on deterministic steady state solutions, $\mathrm{V}$ and $\xi$.

\section{Chemical oscillator}

The chemical oscillator model known as oregonator was devised by Field and Noyes [30] based on the criticism made by Tyson and Light [31] on the original Brusselator model which is two molecular species reaction model. The modified chemical oscillator model consists of three molecular species, $\mathrm{X}, \mathrm{Y}$ and $\mathrm{Z}$ involved in the following five reaction channels which are given in the Table 2 with reaction rates.

The state vector at any instant of time $\mathbf{t}$ along the stochastic trajectories of the variables of the oregonator reaction model is given by, $\vec{S}(t)=[X(t), Y(t), Z(t)]^{T}$. Following the same procedure, the Master equation of this reaction model is given by,

$$
\begin{aligned}
& \frac{\partial P(\vec{S}, t)}{\partial t}=k_{1} A(Y+1) P(X-1, Y+1, Z ; t)+ \\
& k_{2}(X+1)(Y+1) P(X+1, Y+1, Z ; t) \\
& +k_{3} C(X-1) P(X-1, Y, Z-1 ; t)+ \\
& \frac{1}{2} k_{4} X(X+1) P(X+1, Y, Z ; t) \\
& +k_{5} E(Z+1) P(X, Y-1, Z+1 ; t)-\left[\begin{array}{l}
k_{1} A Y+k_{2} X Y+ \\
k_{3} C X+\frac{1}{2} k_{4} X^{2}+k_{5} E Z
\end{array}\right] P(X, Y, Z ; t)
\end{aligned}
$$

where, $\left\{\mathrm{k}_{\mathrm{i}}\right\}, \mathrm{i}=1,2, \ldots, 5$ are reaction rate constants and A, B, C, D and $\mathrm{E}$ are constants in this model. The corresponding CLE of the oregonator model by approximating Master equation (27) and defining macroscopic variable $\{\vec{x}(t)\}=\frac{1}{V} \vec{S}(t)$ are given by,

$$
\begin{aligned}
& \frac{d x(t)}{d t}=k_{1} A y-k_{2} x y+k_{3} C x-k_{4} x^{2}+\frac{1}{\sqrt{V}} \\
& {\left[\sqrt{k_{1} A y} \xi_{1}-\sqrt{k_{2} x y} \xi_{2}+\sqrt{k_{3} C x} \xi_{3}-\sqrt{k_{4} x^{2}} \xi_{4}\right]} \\
& \frac{d y(t)}{d t}=-k_{1} A y-k_{2} x y+k_{5} E z-\frac{1}{\sqrt{V}} \\
& {\left[\sqrt{k_{1} A y} \xi_{5}+\sqrt{k_{2} x y} \xi_{6}-\sqrt{k_{5} E z} \xi_{7}\right]} \\
& \frac{d z(t)}{d t}=k_{3} C x-k_{5} E z+\frac{1}{\sqrt{V}}\left[\sqrt{k_{3} C x} \xi_{8}-\sqrt{k_{5} E z} \xi_{9}\right]
\end{aligned}
$$

Proceeding in the same way as done in the genetic oscillator case, we calculated the deterministic steady state solutions $(\{\xi\} \rightarrow 0)$ which are given by,

$$
\begin{aligned}
& x_{d}^{*}=\frac{A k_{1}}{2 k_{2}}\left(\sqrt{1+\frac{8 C k_{2} k_{3}}{A k_{1} k_{4}}}-1\right) \\
& y_{d}^{*}=\frac{A k_{1} k_{4}}{4 k_{2}^{2}}\left(\frac{4 C k_{2} k_{3}}{A k_{1} k_{4}}-\sqrt{1+\frac{8 C k_{2} k_{3}}{A k_{1} k_{4}}}\right) \\
& z_{d}^{*}=\frac{A C k_{1} k_{3}}{2 E k_{2} k_{5}}\left(\sqrt{1+\frac{8 C k_{2} k_{3}}{A k_{1} k_{4}}}-1\right)
\end{aligned}
$$

Similarly the steady state solutions in stochastic system are obtained by solving the CLEs (28)-(30) in the same way and are given by,

$$
x_{s}^{*}\left(x_{d}^{*}, \xi_{1}^{\prime}, V\right) \sim U_{1} x_{d}^{*}\left(1+\frac{\xi_{1}^{\prime}}{\sqrt{V}} L\right)
$$

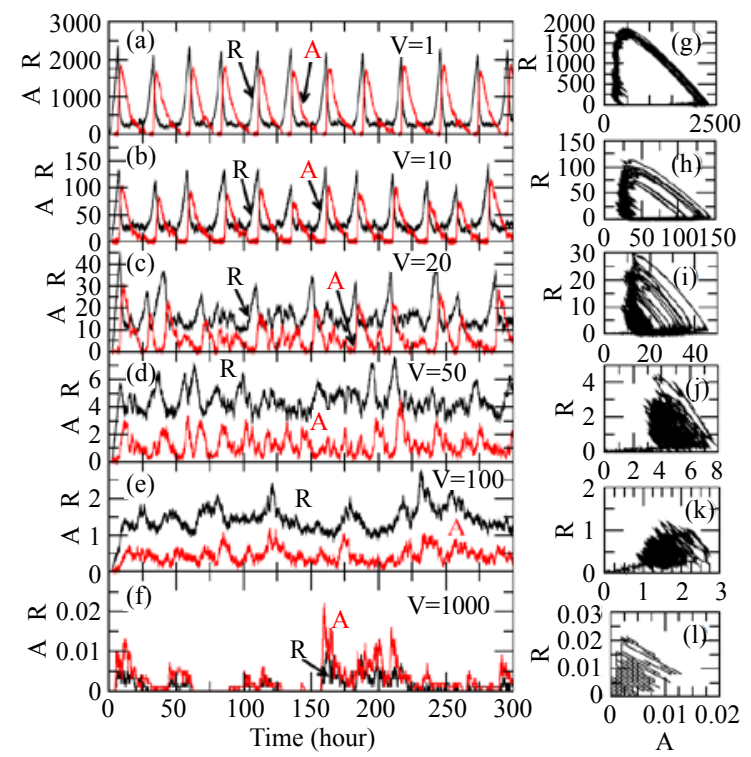

Figure 1: The plots of the dynamics of protein concentrations $A$ and $R$ for different values of system size, $V=1,20,50,100$ and 1000 respectively. The parameter values are taken from Vilar et al. [3] for fixed point oscillation. Panels (a), (b), (c), (d), (e) and (f) show the variations of concentrations of $A$ and $R$ as a function of time in hours for various values of V. Panels (g), (h), (i), (j), (k) and (I) are two dimensional plots of $A$ as a function of $R$ for corresponding values of $\mathrm{V}$. 
Citation: Brojen Singh RK, Indrajit Sharma B (2012) Complexity in the Role of Noise in Stochastic Systems. J Comput Sci Syst Biol 5: 016-023. doi:10.4172/jcsb.1000086

$$
\begin{aligned}
& y_{s}^{*}\left(y_{d}^{*}, \xi_{2}^{\prime}, V\right) \sim U_{2} y_{d}^{*}\left[1+\frac{\xi_{2}^{\prime}}{\sqrt{V}}\left(\frac{1}{2} L+\frac{1}{9 x_{d}^{*}} \sqrt{\frac{k_{4}}{V}}\right)\right] \\
& z_{s}^{*}\left(z_{d}^{*}, \xi_{3}^{\prime}, V\right) \sim U_{3} z_{d}^{*}\left[1+\frac{\xi_{3}^{\prime}}{\sqrt{V}}\left(L+\frac{1}{\sqrt{3 C k_{3} x_{d}^{*}}}\right)\right]
\end{aligned}
$$

where, the vector of proportionality constants for this oregonator model is given by, $\vec{U}=\left(U_{1}, U_{2}, U_{3}\right)^{T}=\left(3,18, \frac{3}{2}\right)^{T}$. The constant $\mathrm{L}$ is given by, $=\frac{1+e x_{d}^{*}}{f}-\frac{r}{1+e x_{d}^{*}}$, where $\mathbf{f}$, $\mathbf{e}$ and $\mathbf{r}$ are also constants given by, $f=6 \sqrt{k_{4}}, e=\frac{2 k_{2}}{A k_{1}}$ and $r=\frac{8 C k_{2} k_{3}}{9 A k_{1} k_{4}}\left(\frac{\sqrt{k_{4}}}{2 C k_{3}}+\frac{2 k_{2}}{3 A k_{1} \sqrt{k_{4}}}\right)$.
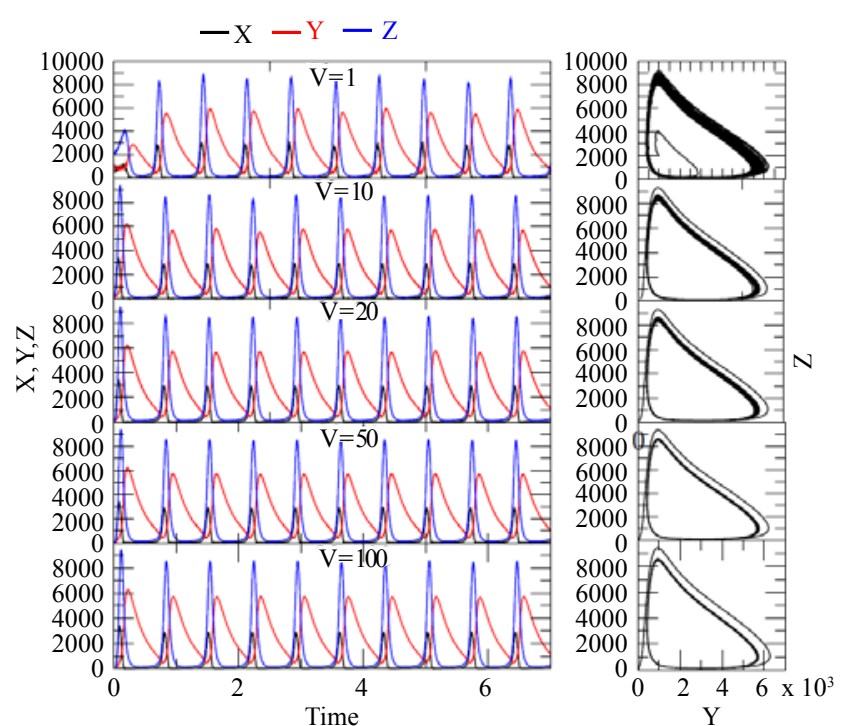

Figure 2: The plots of time evolution of the populations of $X, Y$ and $Z$ for various values of system sizes, $V=1,10,20,50,100$. The right panels are corresponding two dimensional plots of the dynamical behaviors.

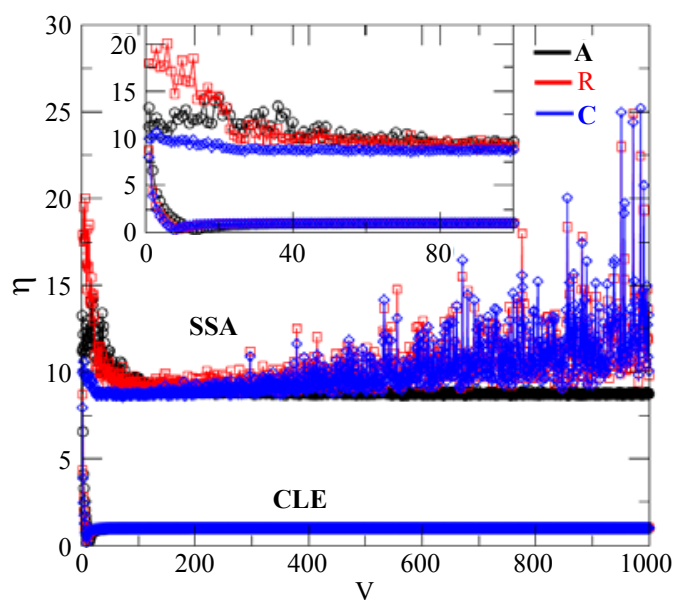

Figure 3: The variation of noise parameter $\eta$ as a function of $V$ in genetic oscillator model calculated by using stochastic simulation algorithm indicated by SSA and using chemical Langevin equations indicated by CLE for A, R and $C$ respectively.

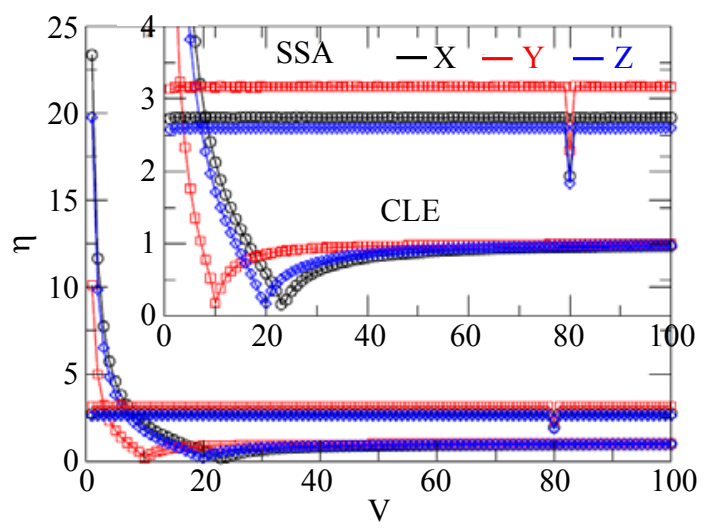

Figure 4: The plots of noise parameter $\eta$ as a function of $V$ for species $X, Y$ and $Z$ in chemical oscillator calculated by stochastic simulation algorithm and chemical Langevin equation indicated by SSA and CLE respectively.

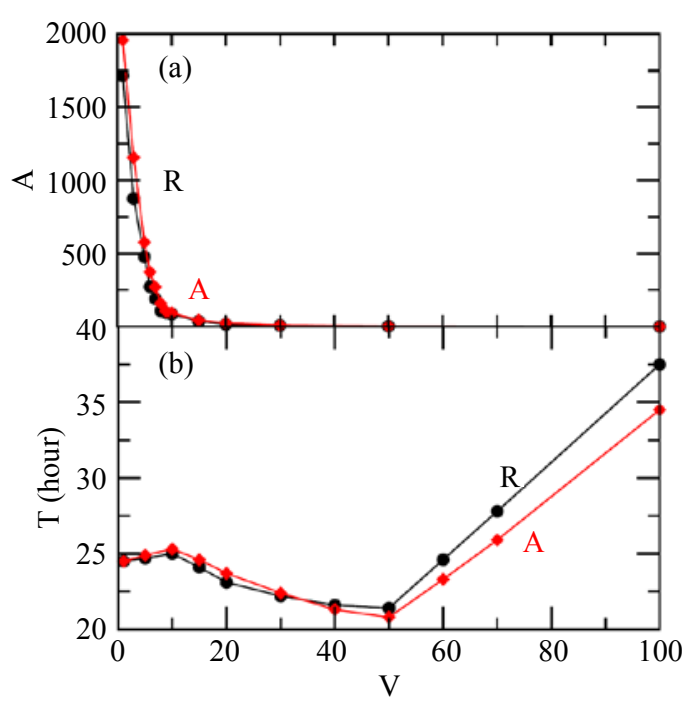

Figure 5: (a) The plot of amplitude Ac as a function of $V$ for $A$ and R. (b) Plot showing the variation of time period $T$ of $A$ and $R$ with respect to $V$.

The two examples studied before indicate that $\vec{U} \sim$ constant for steady state solutions with approximations upto of the order of $0\left(\xi^{2}\right)$. This could lead the CLE in equation (3) and dynamics of noise in equation (5) to the following expression,

$$
\begin{aligned}
& \frac{d \vec{x}_{i}^{s}(t)}{d t} \sim U_{i} F_{i}^{d}\left[\omega\left(\boldsymbol{x}^{d}\right), v\right]+G_{i}\left[\omega\left(\vec{x}^{s}\right), v, \mathbf{V}\right] \xi \\
& \frac{d \vec{\eta}_{i}(t)}{d t} \sim G_{i}\left[\omega\left(\vec{x}^{s}\right), v, \mathbf{V}\right] \xi
\end{aligned}
$$

We now present the stochastic simulation results of the biochemical reaction network of genetic oscillator for different values of system sizes, V by using SSA [1] and are shown in Figure 1. The results show that as $\mathrm{V}$ increases the fluctuation due to noise in the dynamics of $\mathrm{A}$ and $\mathrm{R}$ increases comparatively as shown in the figure. The populations of $\mathrm{A}$ and $\mathrm{R}$ reduce monotonically and the oscillatory behavior start vanishing as $\mathrm{V}$ increases as shown in panels Figure 1 (a)-(f). The panels Figure 1 (g)-(l) show the two dimensional plots of A and $\mathrm{R}$ indicate the loosing of oscillatory behavior as $\mathrm{V}$ increases as the system goes from stochastic 
Citation: Brojen Singh RK, Indrajit Sharma B (2012) Complexity in the Role of Noise in Stochastic Systems. J Comput Sci Syst Biol 5: 016-023. doi:10.4172/jcsb.1000086

to deterministic regime, which is in agreement with earlier results [28]. We could see the drastic decrease in amplitude, destruction of 24 hour period and comparative increase in noise fluctuation as $\mathrm{V}$ increases. The dynamics of the variables tend to maintain least fluctuated sustain oscillation as $\mathrm{V}$ decreases ( $\mathrm{V} \leq 13 \pm 3$ ), above this the oscillatory behavior start getting destroyed and get transition from fluctuated to limit cycle oscillation to no oscillation regime as $\rightarrow \infty$.

Similar results of temporal dynamics of $\mathrm{X}, \mathrm{Y}$ and $\mathrm{Z}$ as a function of $\mathrm{V}$ for chemical oscillator which are shown in Figure 2. The left panels show the decrease in fluctuation in amplitude of oscillation of the variables and thickness in $2 \mathrm{D}$ plots in right panels of Figure 2 as $\mathrm{V}$ increases. The noise fluctuation becomes minimized around $\mathrm{V} \sim 50 \pm 5$ and the oscillatory behavior goes to minimized limit cycle oscillation as shown in $2 \mathrm{D}$ plots in the right panels of Figure 2.

We next estimate the noise in the variable dynamics as a function of $\mathrm{V}$ by using the expression $\eta=\frac{\sigma}{m}$, where $m=\left\langle X_{i}\right\rangle,\left\{\mathrm{X}_{\mathrm{i}}\right\}=\{A, R, C, X, Y, Z\}$, is the mean with $\langle\ldots$.$\rangle as time average and \sigma=\sqrt{\left\langle X_{i}^{2}\right\rangle-\left\langle X_{i}\right\rangle^{2}}$, is the standard deviation. We calculated $\eta$ for stochastic dynamics obtained by both SSA and CLE and time averaging between (50-300) hours which are shown in Figure 3 for genetic oscillator and Figure 4 for chemical oscillator respectively. In the genetic oscillator case, $\eta$ for A, R and C in SSA are found to be decreased as V increases in the interval (1-145) hours, however $\eta$ for $\mathrm{R}$ and $\mathrm{C}$ start increasing as a function of $\mathrm{V}$ for $\mathrm{V}>$ 145 hours, but $\eta$ for A remains almost constant. This behavior of noise might help in maintaining oscillatory behavior in stochastic regime. However, in the case of CLE, $\eta$ decreases as $\mathrm{V}$ increases till $\mathrm{V} \sim 8,10$ and 12 hours for $\mathrm{C}, \mathrm{R}$ and $\mathrm{A}$ respectively; then $\eta$ remains constant as $\mathrm{V}$ increases. The $\eta$ s calculated using SSA for all variables are found to be larger than those calculated using CLE.
Similar calculation of $\eta$ for chemical oscillator is done and the results are shown in Figure 4. The results of $s$ of X, Y and Z due to SSA shows slow decrease in $\eta$ as a function of $V(1<V<50)$ then remain constant. The magnitude of $\eta$ for $\mathrm{Y}$ is largest and that of $\mathrm{Z}$ is smallest. The $\eta$ calculated using CLE for all variables are found to be smaller than those of the calculated using SSA.

The enormously decrease in amplitude of $\mathrm{A}$ and $\mathrm{R}$ are shown in Figure 5 (i). At the same time destruction of 24 hour period of oscillation of $A$ and $R$ as the function of $V$ is shown in Figure 5 (ii). We also obtain that for large $\mathrm{V}(\mathrm{V}>13 \pm 3)$ the amplitudes become getting small and random in nature. However the time period becomes getting large and random in nature for large $\mathrm{V}(\mathrm{V}>13 \pm 3)$. This shows the destruction of rhythmic behavior for large $\mathrm{V}$. The reason may be due to the noise which could able to maintain the rhythmic behavior in the dynamics of $\mathrm{A}$ and $\mathrm{R}$ in stochastic system.

The results of amplitudes and period of oscillations for $\mathrm{X}, \mathrm{Y}$ and $\mathrm{Z}$ are different for chemical oscillator as shown in Figure 6. The amplitudes of these variables $\mathrm{X}, \mathrm{Y}$ and $\mathrm{Z}$ found to slowly increasing till $\mathrm{V} \leq 50 \pm 4$, then these amplitudes found to remain constant for $\mathrm{V}>50$. The time periods of these variables X $(\sim 0.51)$, Y $(\sim 0.48)$ and Z $(\sim 0.49)$ are found to be not much vary with $\mathrm{V}$, but increase slowly till $\mathrm{V} \leq 50 \pm 4$ and remain constant for $\mathrm{V}>50$.

The destructing or maintaining oscillatory behaviors of the variables as a function of system size could be mainly due to noise associated with the variables. The noise associated with the variables in genetic oscillator is found to be constructive in small system size limit (stochastic regime) which could maintain oscillatory behavior and destruct this oscillatory pattern at large system size limit (deterministic regime). The transition from fluctuated limit cycle limit to no oscillation limit as $\mathrm{V} \rightarrow \infty$ supports this claim in genetic oscillator model.
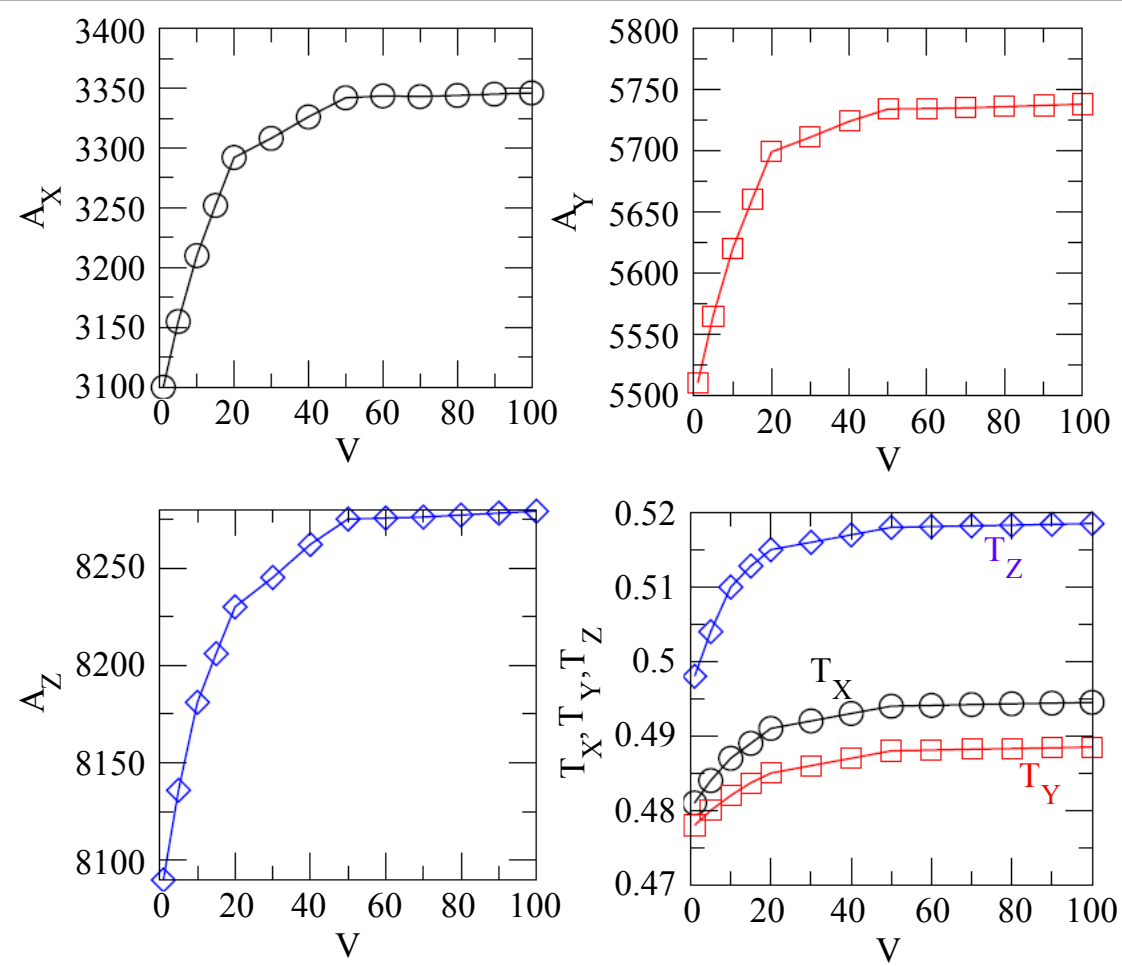

Figure 6: (a) The plot of amplitude $A c$ as a function of $V$ for $A$ and $R$. (b) Plot showing the variation of time period $T$ of $A$ and $R$ with respect to $V$. 
Citation: Brojen Singh RK, Indrajit Sharma B (2012) Complexity in the Role of Noise in Stochastic Systems. J Comput Sci Syst Biol 5: 016-023. doi:10.4172/jcsb.1000086

The opposite function of noise, as we found in genetic oscillator, is obtained in chemical oscillator case supported by the transition from fluctuated limit cycle to normal (minimized fluctuations) limit cycle (sustain oscillation) as $\mathrm{V} \rightarrow \infty$. This shows that the role of noise depends on different factors, for example topology of the network, the way how the molecules interact in the network, system size, strength of the noise associated with the variables etc.

\section{Conclusion}

The calculation of steady state solutions of the noise free functions of all variables in CLE is found to be proportional to the deterministic steady state solutions. The function $\vec{U}$ is found to be constant in the two oscillators but with different values. The noise term in CLE is comparatively small as compared to that in Master equation.

The behavior of noise is found to be different in different systems and depends on various parameters such as topology of the network, system size etc. The noise in genetic oscillator is found to be constructive to maintain oscillatory behavior in stochastic system but destructive in deterministic system by destructing oscillation. This role of noise in chemical oscillator is found to be contrast as compare to genetic oscillator i.e. noise in stochastic system fluctuates the dynamics of the variables tending to destruct the oscillatory behavior, whereas in deterministic regime the oscillatory behavior is maintained stable. There are different interesting issues to be studied in such systems such as phenomenon of stochastic resonance, switching behavior in biological systems, means of inter-oscillator communication etc. These studied could able to highlight different interesting roles of noise in different stochastic systems, especially in biological systems.

\section{Acknowledgments}

We would like to thank Prof. R. Ramaswamy for his valuable suggestions and discussions. This work is financially supported by Department of Science and Technology (DST) in fast track scheme and carried out in Center for Interdisciplinary Research in Basic Sciences, Jamia Millia Islamia, New Delhi, India.

\section{References}

1. Gillespie DT (1977) Exact stochastic simulation of coupled chemical reactions. J Phys Chem 81: 2340-2361.

2. Rao CV, Wolf DM, Arkin AP (2002) Control, exploitation and tolerance of intracellular noise. Nature (London) 420: 231-237.

3. Swain PS, Elowitz MB, Siggia ED (2002) Intrinsic and extrinsic contributions to stochasticity in gene expression. Proc Nat Acad Sci USA 99: 12795-12800.

4. Paulsson J (2004) Summing up the noise in gene networks. Nature (London) 427: 415-418.

5. Panter P (1965) Modulation, Noise and Spectral Analysis, McGrawHill, New York.

6. Anishchenko VS, Neiman AB, Moss F, Schimansky-Geier L (1999) Stochastic resonance: noise-enhanced order. Physcs-Uspekhi 42: 7-36.

7. Hanggi $P$ (2002) Stochastic resonance in biology. How noise can enhance detection of weak signals and help improve biological information processing. Chemphyschem 12: 285-290.

8. Blake WJ, Kaern M, Cantor CR, Collins JJ (2003) Noise in eukaryotic gene expression. Nature 422: 633-637.

9. Becskei A, Seraphin B, Serrano L (2001) Positive feedback in eukaryotic gene networks: cell differentiation by graded to binary response conversion. EMBO J 20: $2528-2535$

10. Weinberger LS, Burnett JC, Toettcher JE, Arkin AP, Schaffer DV (2005) Stochastic gene expression in a lentiviral positive-feedback loop: HIV-1 Tat fluctuations drive phenotypic diversity. Cell 122: 169-182.

11. Kussell E, Leibler S (2005) Phenotypic diversity, population growth, and information in fluctuating environments. Science 309: 2075-2078.
12. Ditzinger T, Ning CZ, Haken H (1993) Stochastic resonance without external periodic force. Phys Rev Lett 71: 807-810.

13. D. Hong, W. M. Saidel, S. Man, J. V. Martin, J. Theor. Biol. 245, 726-736 (2007).

14. R.K. Brojen Singh, Vikram Singh and Ram Ramaswamy, J. Syst. Sc. \& Complx 23, 978 (2010).

15. Zhou C, Kurths J (2002) Noise-induced phase synchronization and synchronization transitions in chaotic oscillators. Phys Rev Lett 88: 230602.

16. Bjørnstad ON, Ims RA, Lambin X (1999) Spatial population dynamics: analyzing patterns and processes of population synchrony. Trends Ecol Evol 14: 427432.

17. Bassler BL (1999) How bacteria talk to each other: regulation of gene expression by quorum sensing. Curr Opin Microbiol 2: 582-587.

18. McQuarrie DA (1967) Stochastic Approach to Chemical Kinetics. J Appl Probab 4: 413-478.

19. Van Kampen (2007) Stochastic processes in Physics and Chemistry, NorthHolland, $3^{\text {rd }}$ Edition.

20. Gillespie DT (2000) The chemical Langevin equation. J Chem Phys 113: 297 306

21. Rosenblum MG, Pikovsky AS, Kurths J (1996) Phase synchronization of chaotic oscillators. Phys Rev Lett 76: 1804-1807.

22. Ramaswamy R, Sbalzarini IF, Nelido GS (2011) Noise-Induced Modulation of the Relaxation Kinetics around a Non-Equilibrium Steady State of Non-Linear Chemical Reaction Networks. PLoS One 6: e16045.

23. Scott M, Ingalls B, Kaern M (2006) Estimations of intrinsic and extrinsic noise in models of nonlinear genetic networks. Chaos 16: 026107-026122.

24. Gillespie DT (2009) Deterministic limit of stochastic chemical kinetics. J Phys Chem B 113: 1640-1644

25. Gillespie DT (2007) Stochastic simulation of chemical kinetics. Annu Rev Phys Chem 58: 35-55.

26. Grima R, Thomas P, Straube AV (2011) How accurate are the nonlinear chemical Fokker-Planck and chemical Langevin equations? J Chem Phys 135 084103.

27. Matias MA, Guemez J (1995) J Chem Phys 102: 1598

28. Vilar JMG, Kueh HY, Barkai N, Leibler S (2002) Mechanisms of noiseresistance in genetic oscillators. Proc Nat Acad Sci USA 99: 5988-5992.

29. Barkai N, Leibler S (2000) Circadian clocks limited by noise. Nature 403: 267268.

30. Field RJ, Noyes RM (1973) J Chem Phys 60, 1877.

31. Tyson JJJ (1973) Chem Phys 58: 3919. Tyson JJ, Light JCJ (1973) Chem Phys 59: 4164. 